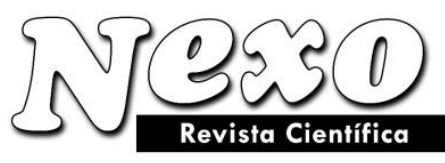

Vol. 34, No. 02, pp. 583-595/Junio 2021
ISSN-E 1995-9516

Universidad Nacional de Ingeniería COPYRIGHT ๑ (UNI). TODOS LOS DERECHOS RESERVADOS

http://revistas.uni.edu.ni/index.php/Nexo https://doi.org/10.5377/nexo.v34i02.11543

\title{
Formación de estudiantes investigadores en ingenierías: acercamiento a su estado del arte
}

\section{Training of engineering research students: approach to the state of the art}

\author{
Rosario Carvajal-Hernández *, Verónica Guerrero-Hernández, Erick de Jesús Téllez-Vera \\ Tecnológico Nacional de México / Instituto Tecnológico Superior de San Andrés Tuxtla. División de \\ Ingeniería Informática. San Andrés Tuxtla, Veracruz, México. \\ *ch.rosario@gmail.com
}

(recibido/received: 07-diciembre-2020; aceptado/accepted: 08-febrero-2021)

\section{RESUMEN}

La actual revisión de la literatura tiene como objetivo identificar la situación actual que presenta la investigación en el entorno de las ingenierías, específicamente en la formación de esta competencia en los estudiantes de educación superior en México abarcando desde el proceso educativo hasta el investigativo, con la finalidad de notificar los avances que se tienen en este país, ya que tales situaciones influyen directamente en el desarrollo de múltiples factores. A través de una revisión documental, se ahondo en los métodos y formas en las que se intenta enseñar la competencia de la investigación en los jóvenes universitarios en América Latina, para posteriormente contrastar su realidad con la de México. Lo anterior posibilitó conocer las opiniones y avances que se ha logrado en el ámbito de la investigación, particularmente en el desarrollo de la competencia investigativa en las Instituciones de Educación Superior en la zona de estudio, así como los actores involucrados en esta noble labor de generar y difundir el conocimiento. Finalmente, la enseñanza para saber investigar precisa del desarrollo de capacidades, análisis crítico, deber ético y compromiso con la sociedad que permitan dejar en claro los requerimientos y situaciones adversas que presenta la educación en este momento.

Palabras claves: estudiantes, ingeniería, investigación, formación, semilleros.

\begin{abstract}
The current literature review aims to identify the current situation presented by research in the engineering environment, specifically in the formation of this competence in higher education students in Mexico, ranging from the educational process to the investigative, with the purpose of reporting the progress made in this country, since such situations directly influence the development of multiple factors. Through a documentary review, it delved into the methods and ways in which it is tried to teach the competence of research in young university students in Latin America, to later contrast its reality with that of Mexico. The foregoing made it possible to know the opinions and progress that have been achieved in the field of research, particularly in the development of investigative competence in Higher Education Institutions in the study area, as well as the actors involved in this noble task of generating and spread knowledge. Finally, teaching to know how to investigate requires the development of capacities, critical analysis, ethical duty and commitment to society that make it possible to clarify the requirements and adverse situations that education currently has.
\end{abstract}


Keywords: students, engineering, research, training, seedbeds.

\section{INTRODUCCIÓN}

En la actualidad la investigación es uno de los ejes principales en la formación de los estudiantes universitarios en el área de ingeniería. El objetivo principal del presente artículo es identificar la situación actual que presenta la competencia en investigación en el contexto de las ingenierías.

Bolatovna et al., (2016) en su trabajo “Forming Master's Degree Students' ICT Competencies as Future Researchers and Educators: a Kazakhstan Case Study" en este año el mundo está en constante cambio en todos los ambientes, abarcando el de la educación, y hablando precisamente de ello, la educación superior, las universidades, los institutos tecnológicos, las universidades tecnológicas y todas aquella que se dedican a esta labor, están comprometidos con el trabajo sustantivo de robustecer la integración de la investigación como eje central de los procesos educativos.

En las Instituciones de Educación Superior (IES) constantemente se habla de la palabra investigación, asumiendo que en los niveles educativos anteriores se aprendió a realizar tan importante actividad. Para González (2019) en su artículo "Alternativas para la formación de investigadores en la educación" indica que se aprende a investigar, investigando. Indudablemente esta aseveración muestra que la actividad requiere una formación previa para que permita generar saberes y capacidad para afrontarla correctamente, así también implica ética y compromiso social que redunden en el beneficio de la sociedad.

En palabras de Pérez (2017) la investigación es: un conjunto de concepciones y dispositivos que se generan y ponen en juego para que una persona o grupo se apropie de un capital académico y cultural; y desarrolle una serie de habilidades, destrezas o competencias propias del oficio de la investigación, con el propósito de producir conocimiento original o aplicar el existente para atender las problemáticas sociales y disciplinarias de un campo en particular.

De acuerdo con Aldana y Castro (2017) en su escrito llamado "Convergencias y divergencias en la apropiación de la formación investigativa: el caso de dos programas académicos colombianos" hacen notar que la investigación es una función inevitable de la educación superior en conjunto con la docencia y la proyección social, dado la contribución al desarrollo de las disciplinas y la solución de problemas, puesto que potencializa las capacidades metacognitivas que permiten aprender a aprender, remarcando que sin formarse en la investigación es inadecuado dialogar de educación superior.

En palabras de Martínez et al., (2018) en su publicación nombrada "Desarrollo de competencias investigativas en los estudiantes", uno de los desafíos primordiales de la educación superior es preparar a profesionales capaces y comprometidos con el desarrollo de la sociedad, innovando soluciones empleando el método científico. Para conseguir lo anteriormente dicho precisa generar competencias investigativas en el alumnado permitiendo gestar un aprendizaje significativo puesto que están cara a cara buscando la solución de problemas, esto conlleva a que construya su conocimiento científico o a reedificarlo. La investigación está concebida como una actividad primordial de las IES actuales.

Hace unos pocos años, para efectuar una investigación, se acudía a una biblioteca convencional, en la que existente incluso todavía un proceso para llevar a cabo la consulta de libros, revistas, tesis o cualquier documento formal que le permite adquirir la información formal que se requiera. Sin embargo, de acuerdo con Ramírez (2015) en su artículo nombrado "Ansiedad bibliotecaria en estudiantes universitarios" indica que los usuarios de dichas bibliotecas expresan "sentirse perdidos" por aspectos tales como: tamaño del lugar, falta de ubicación de las cosas, cómo iniciar y qué realizar. 
Actualmente la realidad con respecto a la consulta de información formal ha cambiado, desde la aparición del Internet, el uso de las computadoras y las Tecnologías de Información y Comunicación (TIC's). De hecho, George y Salado (2019) en su publicación "Competencias investigativas con el uso de las TIC en estudiantes de doctorado" manifiestan que existen evidencias que demuestran que los alumnos utilizan con frecuencia las TIC's, y poseen la facilidad de verificar la formalidad de la información que obtienen de Internet.

Troncoso et al., (2014) en su trabajo llamado "Cómo transformar al alumno en investigador" reconocen que el binomio conocimientos-habilidades permiten en la persona originar, divulgar y emplear dicho conocimiento en su propia vida en las diversas facetas que esta posee, logrando con ello incrementar su productividad y calidad de vida. En México, entre los retos a los que se enfrentan las IES está el generar habilidades de investigación en su matrícula educativa, ya que aún persiste la diferencia entre el número de egresados y titulados. De acuerdo con la Asociación Nacional de Universidades e Instituciones de Educación Superior (ANUIES) en un informe Educación Superior indican que los egresados representan un $64 \%$ y los titulados $39 \%$, lo que representa una diferencia de $25 \%$.

Acorde con Acuña Gamboa (2018) en la publicación "Formación de investigadores educativos en Chiapas: realidades y falacias" externa que en uno de los dictámenes mostrados en el Plan Nacional de Desarrollo (PND) 2013-2018 evidencia que México está en la lista de aquellos países que aportan en menores cantidades a la generación del conocimiento, abajo del 1\% del total alcanzado a nivel mundial.

Además, conforme con los datos publicados por el Banco Mundial (2020) en su informe del "Gasto en investigación y desarrollo (\% del PIB)" en donde notifica los porcentajes que cada país invierte en este rubro tan importante en el desarrollo y por ende en la calidad de vida de sus habitantes, en el que se visualiza que el porcentaje que destina México a Investigación y Desarrollo (I+D), la cifra que se tiene oficialmente hasta el 2018 es de 0,31\% del PIB. Esta cifra es muy similar a la aportada en países como en Nepal en 2010 con el 0,30\%, Mozambique en 2015 con el 0,34\% y Chile en 2017 con 0,36\%. En contraparte se encuentran los países que le aportan un porcentaje alto, teniendo en los primeros lugares del 2018 a Alemania con $3,09 \%$, Japón con 3,26 e Israel con $4.95 \%$.

González (2019) en la publicación "La formación de jóvenes investigadores en Alemania y México, un estudio comparativo" exponen que, en años recientes, México le ha resultado atractivo a las empresas tecnológicas, centros de investigación y universidades internacionales. A partir de lo cual, el país conseguirá un inmejorable desarrollo si puede ubicar a la población y en especial a los alumnos que están próximos a cursar una carrera como gestores, diseñadores, desarrolladores y profesionales con una formación consolidada en ambientes como la investigación científica, tecnológica y la innovación. Por otro lado, se debe mencionar que en Latinoamérica está presente un atraso extraordinario con respecto a la cantidad de investigadores, y por ende la formación de profesionales en este aspecto.

Campos y Ramírez (2018) en su artículo "Las TIC en los procesos educativos de un centro público de investigación" expresan que en caso de que los alumnos logren conseguir estas competencias, desarrollaran con más precisión actividades de índole científico y les permitirán divulgar los resultados de tales trabajos de investigación, afianzando la investigación en su institución educativa.

Parra-Castrillón (2018) declarara en su publicación "Construcción de la competencia investigativa en ingeniería" que la construcción de la competencia investigativa en los alumnos dentro de las instituciones de educación superior es todo un desafío, puesto que hay que involucrar a tales alumnos en la participación de proyectos y procesos de investigación, con la responsabilidad que ello requiere. En las ingenierías se presentan dos momentos en los que se puede acercar a los alumnos a la investigación, el inicial en los primeros semestres debido a que los alumnos están interactuando con la experimentación, las matemáticas; el segundo momento por el carácter del perfil de los ingenieros al estar asociado con la innovación y el 
desarrollo tecnológico, cuestiones que reclaman postura, acciones y saberes en la investigación formativa. Así mismo, este autor define a la competencia investigativa como la integración de conceptos, actitudes y procedimientos que demuestra un investigador en la planeación, desarrollo y divulgación de iniciativas para la generación y gestión de nuevo conocimiento.

\section{DESARROLLO}

Este trabajo se enfoca en efectuar una revisión de la literatura acerca de la investigación y su enseñanza en las IES latinoamericanas, así como un análisis acerca de la enseñanza de las competencias investigativas y las tendencias que se gestan en otros países, las cuales les han permitido elevar su productividad académica.

Se revisaron 100 artículos exhaustivamente, empleando para este trabajo 36 artículos debido a la naturaleza de su información, los cuales fueron obtenidos de bases de datos tales como: Redalyc, Scielo, Google Académico y Elsevier se procedió a plasmar. Estos artículos son de diversos países, entre los que destacan México, Colombia y España. Así mismo, la búsqueda de los artículos está comprendida en un rango de 2015 y 2020, únicamente en idioma español.

Carrasco, Baldivieso y Di Lorenzo (2016) en su primicia nombrada "Formación en investigación educativa en la sociedad digital. Una experiencia innovadora de enseñanza en el nivel superior en el contexto latinoamericano" advierten que en la investigación no existen esquemas o modelos únicos y absolutos, sino que son simples guías que encauzan el desarrollo del trabajo de investigación, mismas que se sincronizan con las necesidades de una situación específica. Aunado a que puede apoyarse de las herramientas tecnológicas de la era digital moderna.

Gómez y García (2015) en el artículo nombrado "Factores influyentes de la gestión del conocimiento en el contexto de la investigación universitaria" afirman que identificaron dos aspectos que retraen la gestión del conocimiento en los equipos dedicados a la investigación, donde el primero de ellos es el tiempo dedicado y el número de personas enfocadas a esta labor. Así también, expresan que la gestión del conocimiento se basa en cuatro pilares: personas, procesos, contenidos y tecnologías de información y comunicación como se aprecia en la Tabla 1.

Tabla 1. Componentes de la gestión del conocimiento

\begin{tabular}{|c|c|}
\hline COMPONENTES & DESCRIPCIÓN \\
\hline Personas & $\begin{array}{l}\text { » Recursos humanos. } \\
\text { » Cultura organizativa interiorizada. }\end{array}$ \\
\hline Procesos & $\begin{array}{l}\text { » Contexto en el que se produce la gestión del } \\
\text { conocimiento. } \\
\text { »Visión y misión de la organización. Estrategias de } \\
\text { negocio. } \\
\text { » Metodología y rutinas organizativas que llevan a cabo las personas. } \\
\text { » Conocimiento asociado a cada área. }\end{array}$ \\
\hline Contenidos & $\begin{array}{l}\text { » Información interna y externa que queda recogida en documentos, bases de } \\
\text { datos, o en las personas. } \\
\text { » Lenguajes documentales controlados. } \\
\text { » Soportes y formatos de presentación (documentos, páginas amarillas, } \\
\text { lecciones aprendidas). }\end{array}$ \\
\hline $\begin{array}{l}\text { Tecnologías de Información y } \\
\text { Comunicación (TIC) }\end{array}$ & $\begin{array}{c}\text { » Medios para recoger, almacenar y distribuir los datos, la información y el } \\
\text { conocimiento explícito y tácito. } \\
\text { » Alineación con la estrategia y las necesidades de la } \\
\text { organización, especialmente las requeridas al estudiar los otros tres } \\
\text { componentes. }\end{array}$ \\
\hline
\end{tabular}


En términos de González (2019) desde la época de los 80's se ha tratado de encontrar otras opciones para facilitar y robustecer la investigación, mediante simposios, foros, debates donde los investigadores con experiencia, docentes investigadores de múltiples IES y alumnos sustancialmente.

De acuerdo con Díaz et. al., (2018) en el artículo "Metodologías e indicadores académicos, económicos, sociales y tecnológicos para la evaluación del impacto de la investigación científica universitaria” expresan que en la educación superior la manera habitual en la que se valora la trascendencia de las investigaciones se ha cimentado en el sondeo de los beneficios académicos mediante técnicas bibliométricas, número de publicaciones, citaciones e ingresos derivados de la investigación. Estos son algunos de los beneficios a los que se alude al realizar investigación, sin evidenciar mejoras en la sociedad, en la economía y la tecnología.

Si bien es cierto que las IES han integrado la formación investigativa en la retícula de los planes de estudio de los programas académicos, que van desde instrucciones en metodología hasta la participación en semilleros, en específico países como Colombia, proyectos de investigación docentes o institucionales, y eventos de esta competencia en mención, no se han generado los resultados anhelados en el aprendizaje de esta capacidad. Lo anterior de acuerdo con Aldana y Castro (2017).

En palabras de Martínez et. al., (2018) el alumno investigador precisa poseer conocimientos de la ciencia de estudio en particular, técnicas y procedimientos para encaminar la investigación, en los pasos iniciales se cuestiona la problemática, en un segundo instante plantea el diseño, analiza los resultados y finalmente genera la conclusión y ofrece sus recomendaciones.

Rojas y Méndez (2017) en la publicación "Procesos de formación en investigación en la Universidad: ¿Qué le queda a los estudiantes?" comentan que la actitud del alumnado con respecto a la investigación cubre 3 consideraciones: la propia trayectoria del alumno incluyendo experiencias y posibilidades, las condiciones institucionales de la educación superior y del desarrollo de sus esquemas de investigación y el impacto lineal de ello en la formación superior.

Pérez (2017), en su trabajo de investigación denominado "Formación para la investigación y eficiencia terminal. Una tensión en los posgrados en Educación" menciona que es necesario que la cultura académica esté enfocada en la apertura a los docentes investigadores, ya que se requiere de condiciones institucionales, consultorías constantes, así como el entorno laboral indicado que permita la noble labor de la investigación.

Aunado a lo anterior, Escalante y García (2016) en el trabajo "La formación de investigadores en el posgrado en México: ¿una tarea solo del docente?" refieren que el docente también tiene un papel en la "formación de investigadores", ya que a través de él se propaga el conocimiento o el aprendizaje. La labor colegiada que realizan los docentes, y en cuyos proyectos participan el alumnado, posibilita que se formen al interactuar en equipos de investigación cuyas actividades sean dirigidas al quehacer científico.

Para Pepper-Loza, y Terán-Vega (2019) en el artículo titulado "El semillero de investigación estudiantil, como estrategia para la Formación de investigadores", comentan la figura alusiva a los semilleros de investigación, indicando el referente histórico de la formación investigativa en la universidad alemana de Humboldt en Berlín, Alemania. Lo anterior es fundamentado en que esta prestigiosa universidad cultiva las ciencias y las artes mediante la investigación, la enseñanza y el estudio profundo de los temas. Basándose en este artículo, los autores expresan al pie de la letra que: "Los semilleros de investigación deberán ser creados cercanos a los Grupos de Investigación formalmente registrados en la respectiva Universidad. La organización interna de los Semilleros de Investigación debe ser de autonomía de cada uno dependiendo del grado de desarrollo, estructuración y consolidación de estos. Sin embargo, es necesario que cuenten con el acompañamiento de un docente-investigador que haga parte del Grupo de Investigación al cual pertenece el 
Semillero, ya sea en calidad de director o subdirector del Semillero. Los jóvenes investigadores estarán vinculados a un Grupo de Investigación, tendrá un tutor asignado y participará en un proyecto de investigación."

Troncoso et. al., (2014) en su trabajo llamado "Cómo transformar al alumno en investigador" expresan asertivamente que las IES en conjunto con sus docentes y responsables tienen la posibilidad de generar programas de investigación institucionales para involucrar al alumno en la investigación, lo que permitirá mejorar su formación integral, la mejora de su lugar, región y país. De hecho, proponen 6 consideraciones del proceso del desarrollo de habilidades de investigación (DHI), las que se muestran en la Tabla 2:

Tabla 2. Consideraciones del proceso de DHI

\begin{tabular}{cc}
\hline No. & Consideración \\
\hline 1 & Entender la investigación como acto de pensar y de actuar consecutivamente \\
2 & Tener la guía y acompañamiento de un profesor que orienta la formación del \\
3 & alumno \\
4 & Profundizar las habilidades de lecto-escritura del alumno \\
5 & Promover en el estudiante la necesidad de pensar y tener curiosidad \\
6 & Uso de herramientas informáticas \\
\hline Fuente: (Troncoso & Motivar la autonomía del alumno, la disciplina y el trabajo constante \\
\hline
\end{tabular}

Fuente: (Troncoso et. al., 2014).

Conforme a lo que indica Estrada et. al., (2017) en la publicación "El docente universitario como promotor del pensamiento crítico, competencia del investigador" el docente precisa generar espacios de aprendizaje para que el alumnado se integre proactivamente en la construcción del proceso de adquisición de habilidades y por ende del conocimiento.

En el mismo tenor de ideas, Pepper-Loza, y Terán-Vega (2019) comentan que los semilleros surgen como una oportunidad precisa en la que los alumnos involucrados en trabajos de investigación, bajo la figura de un investigador, quien funge como tutor, permiten generar conjuntamente, comunidades de aprendizaje en torno a un tema de investigación, de la creación y desarrollo de proyectos, así como la divulgación de los resultados, así como la búsqueda de financiamiento. Una valiosa aportación de los semilleros es que se ensamblan como comunidad académica al sistema de investigación de la Institución, relacionados con los grupos de investigación. Los semilleros deben estar estructurados como mínimo por un docente asesor, un alumno líder y tres alumnos miembros, dichos alumnos deben tener una matrícula vigente.

Para Rojas Silva (2018) en el capítulo "Héroes del futuro: Desafío para la formación de investigadores con sensibilidad social", los semilleros de investigación son escenarios extracurriculares de investigación formativa que potencializan múltiples capacidades académicas y sociales del alumnado, entre las que se encuentran la lectura juiciosa y trabajo colaborativo.

Aunado a lo anterior, Guerrero et. al., (2019) en su artículo "Propuesta de semilleros de investigación para el desarrollo de la competencia investigadora en ingenierías" plantean que los semilleros de investigación permitirán preparar al alumnado en la competencia investigadora en las ingenierías. Lo anterior lo representan en la Figura 1: 


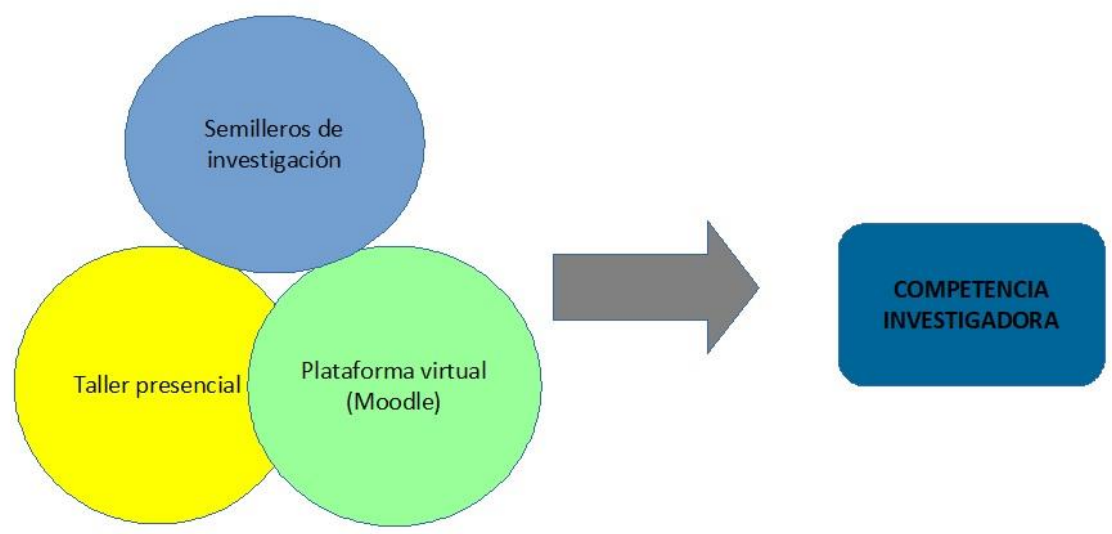

Fuente: Guerrero et. al., (2019)

Figura 1. Propuesta de semilleros de investigación

Actualmente esta modalidad de los semilleros de investigación se desarrolla en el Tecnológico Nacional de México, Campus San Andrés Tuxtla, donde desde el año 2017 se implementa bajo la denominación Programa Institucional de Semilleros de Investigación (PISI). Dado los diversos factores que convergen para efectuar adecuadamente la labor de promover la investigación en la Institución en comento, este programa de semilleros actualmente se encuentra trabajando con una ligera modificación a la figura anterior, como se puede observar a continuación en la Figura 2:

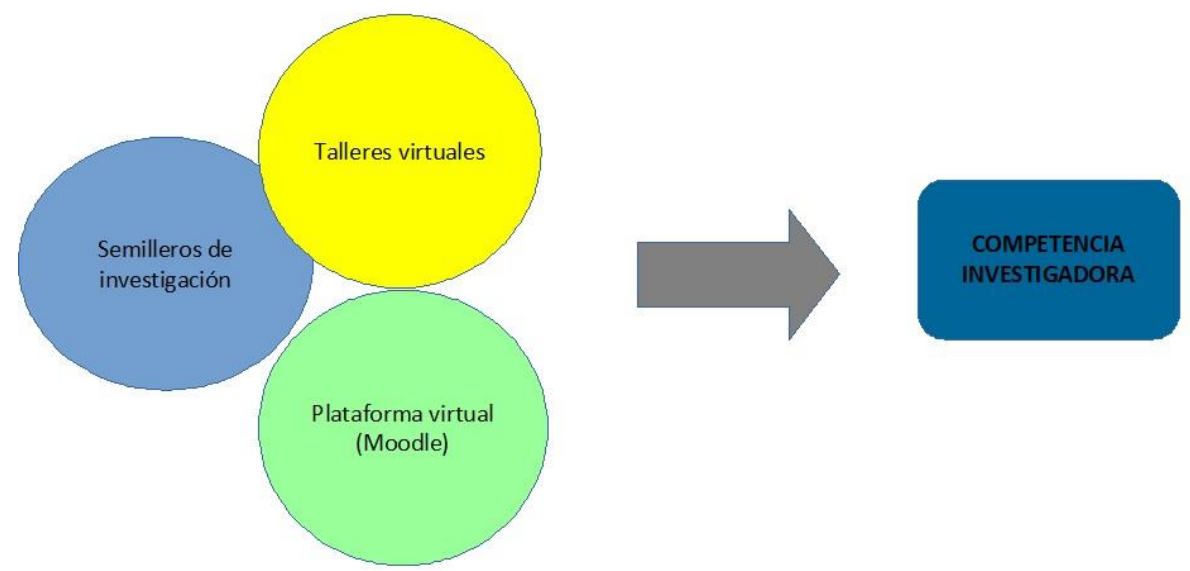

Figura 2. Propuesta de semilleros de investigación virtual Fuente: Elaboración propia

Vega-Monsalve (2019) en su publicación denominada "Estrategias de conformación y consolidación de semilleros de investigación en pregrado. Estudio de caso en una institución de educación superior en Colombia", aclara que existen diversos estudios de múltiples autores y en países diferentes a Colombia y exponen los factores que influyen en el éxito de los equipos de investigación, tales como: compromiso de los estudiantes con el proceso, liderazgo del docente, la dinámica de trabajo por parte de las estrategias que el docente líder genere para conservar la motivación en los participantes del semillero de investigación y apoyo institucional. Lo antes descrito alude a que la dirección exitosa está basada en el compromiso de todos los actores involucrados que van desde la Institución, los docentes y alumnos.

Del mismo modo, Lozoya y Ocampo (2019) en su capítulo de libro: "Estrategias para la formación de investigadores en investigación educativa” retoman la función del docente como guía y facilitador, en un eje fundamental para el desarrollo apropiado en la instrucción del investigador. 
Ruiz (2019) en su artículo publicado llamado "Evaluación formativa y compartida para el desarrollo de competencias investigativas en estudiantes universitarios" resalta el aprendizaje de cuatro competencias requeridas para el crecimiento en la instrucción de la investigación científica: 1) identificación de un objeto de estudio; 2) formulación de preguntas de investigación para resolver problemas; 3) identificación y planteamiento de problemas; y 4) trabajo en equipo.

Por otra parte, Pastor (2020) expresa que las capacidades de investigación se fundamentan en el empleo de entornos virtuales de aprendizaje (EVA), y en base a su trabajo realizado determinaron que son 5 las capacidades de investigación que son primordiales en el proceso de aprendizaje de los alumnos de las IES, 1. Administración de literatura bibliográfica, 2. Búsqueda de información bibliográfica, 3. Procesamiento y obtención de información, 4. Estadística descriptiva e inferencial y 5. Análisis e investigación de proyectos.

Aunado a ello, Cifuentes y Pedraza (2017) en el trabajo denominado "Importancia de la investigación en la formación de estudiantes en la modalidad a distancia" comparten que la modalidad de educación a distancia nace a partir de una contestación a los requerimientos de la juventud, adultos y profesionales que por diversas razones el asistir al sistema escolarizado les resulta imposible. Esta faceta de educación es posible ofertarla en cualquier tiempo y lugar, sin embargo, se considera un reto para que funcione adecuadamente, ya que se requiere que el estudiante posea la capacidad de auto-aprendizaje. Los autores proponen las características de la educación a distancia, las cuales se muestran en la Tabla 3:

Tabla 3. Características de la educación a distancia.

\begin{tabular}{cc}
\hline No. & Característica \\
\hline 1 & Elimina la barrera del espacio y de la distancia \\
2 & Elimina la barrera de la edad y de la ocupación \\
3 & El grupo de estudiantes es heterogéneo \\
4 & Población especialmente dispersa \\
5 & Permite el acceso masivo \\
6 & Facilita el trabajo individual \\
\hline Fuente: Cifuentes, J. y Pedraza, J. (2017)
\end{tabular}

Aunado a lo anterior, Flores (2018) en la publicación "Retos y contradicciones de la formación de investigadores en México" comentan que los tiempos de término de una investigación se especifican por la administración y encaminan a los investigadores y al alumnado participante a efectuar las actividades condicionados por el tiempo y no por la solución de problemas.

En ese contexto, ante la actual pandemia-sindemia que se presenta en el mundo, la modalidad a distancia, empleando plataformas virtuales, permite formar investigadores facilitándoles los recursos educativos para que puedan ser capaces de adquirir las competencias investigativas y mediante ello mejorar su desempeño académico, profesional y personal.

Cruz et al., (2019) muestran en el trabajo "Las Tecnologías de la Información y de la Comunicación (TIC) como forma investigativa interdisciplinaria con un enfoque intercultural para el proceso de formación estudiantil" que las TIC se generan de aquellos avances científicos originados en el ámbito de la informática y de las telecomunicaciones, es por ellos la trascendencia de la tecnología que interviene en el procesos de producción, interacción, tratamiento y comunicación de la información.

Landeros-Olvera et. al., (2018) en el artículo "Rompiendo paradigmas en la formación de jóvenes investigadores estudiantes de enfermería" expresan certeramente que "La investigación se enseña haciendo 
investigación", ya que esta es la manera para empoderar a los alumnos que cursan una licenciatura a instruirse como científicos. Es necesario, por lo tanto, tratarlos como investigadores.

\section{DISCUSIÓN}

Las capacidades que el alumno va desarrollando a lo largo de su aprendizaje de cómo hacer investigación le añade vivencias formativas para los diversos ámbitos de su vida personal y profesional. Es preciso que tanto los docentes como alumnos consideren a la investigación como parte de la retícula, actualmente en los planes de estudios de las diversas carreras que se imparten en el Tecnológico Nacional de México se encuentran tres materias, sin embargo, todavía se consideran como asignaturas aisladas a las demás, cuando en realidad tienen igual importancia o más que otras debido a la trascendencia que pueden brindarle a los diversos entes que están involucrados, que va desde el alumno, el docente, la institución y finalmente la sociedad.

Las IES requieren transformarse en el recinto que fomente la investigación para el desarrollo de las competencias investigativas viéndose como un proceso que se genera desde el comienzo de este nivel educativo, esto de acuerdo con Martínez et. al., (2018) ya que son los primeros semestres en los que se puede encaminar esta competencia para lograr mejores resultados en sus estudios, aunado a que es oportuno motivar a los alumnos en la investigación puesto que mediante ella se pueden resolver múltiples problemáticas que nos afectan como sociedad, cultura y país; puesto que tienen la posibilidad de integrarse a proyectos, líneas de investigación, cuerpos académicos en los que pueden desarrollarse y consolidarse en esta competencia.

Pedraza (2018) en su artículo "Experiencias de formación como investigadores educativos de estudiantes de un programa de doctorado en educación" afirma que el hecho de instruir investigadores es una labor ardua, que puede transformarse en un reto para las IES y para los facilitadores. Su complejidad va más allá de los aspectos académicos y las estrategias institucionales utilizadas; es idóneo considerar la calidad con la que se integra al proceso formativo del alumnado. De igual forma, Troncoso et. al., (2014) en su trabajo llamado "Cómo transformar al alumno en investigador" debaten sobre la imperativa urgencia con respecto a que los estudiantes generen habilidades de investigación, y por ende la divulgación de resultados en cualquier medio formal que permita compartir los conocimientos generados.

Finalmente, retomando palabras de Vega-Monsalve (2019), las IES tienen varios retos en la consolidación de este tipo de estrategias: crear formas de estímulo y retención de los estudiantes una vez se gradúan y egresan de la institución, asignar horas en los planes de trabajo de los docentes que lideran los semilleros y aportar los recursos para su funcionamiento, diseñar un perfil especial para los docentes que se encargarán de esta labor, reconociendo que no solo es necesario que cuente con formación y experiencia investigativa, sino que también disfruten digiriendo estudiantes y transmitiendo sus conocimientos, planear una forma de evaluación y seguimiento que no desconozca la naturaleza formativa y no productiva de los sistemas de investigación, e integrar esta experiencia con los cursos que dentro del currículo ofrecen formación investigativa.

Jiménez (2018) en el mensaje de la editorial de la Revista de Investigación en Ciencias Sociales y Humanidades expresa que aquellos que laboran en el área de la investigación, así como en la formación de alumnos, comprenden que el hecho de formar investigadores no se efectúa en corto tiempo, ya que es un proceso. El alumno que desarrolla la pasión por la investigación tendrá un proceso de formación distinto, contarán con la mirada juiciosa y entenderán aspectos que otros no logran percibir.

Olivares et al., (2016) previenen en la publicación "Las TIC en educación: metaanálisis sobre investigación y líneas emergentes en México" de la premura en efectuar más investigación enfocada a la gestión y calidad 
de programas educativos, la virtualización de la educación, niveles, modelos y modalidades de educación mediante tecnologías y estudios enfocados a política educativa y cambio social.

\section{CONCLUSIONES}

Las tecnologías de la información y comunicación (TIC's) son herramientas clave en diversos ámbitos de la sociedad, pues su creciente campo de aplicación y las oportunidades que ofrece en términos de innovación en la época en que vivimos son bastante amplias. Es menester reconocer que nuestras vidas están ligadas de manera cada vez mas fuerte al uso de estas tecnológicas. Por ejemplo, en el sector educativo y de investigación, las TIC's fomentan la colaboración y propician el acceso a una cantidad vasta de fuentes de consulta sin precedentes. Sus facilidades son un atractivo, ademas, para inducir a los estudiantes a ingresar al mundo de la investigación. No obstante, las herramientas por si mismas carecen de un uso significativo si quienes las utilizan carecen del conjunto necesario de actitudes y aptitudes que les permitan lograr sus objetivos, pudiendo ser estos formativos o de investigación.

Ciertamente, las palabras de Durand-Villalobos (2017) en el artículo denominado "Factores que inciden en el desempeño de los grupos de investigación: tres casos de estudio de la Universidad de Sonora" expresan que los equipos de investigación están en camino de desarrollo, sin embargo, para afianzar los resultados, se requiere que la Institución admita la valía de las capacidades de aquellos que realizan investigación y estimule la generación de nuevos conocimientos. Los equipos de investigación precisan dominar las técnicas del trabajo colaborativo y mejorar las destrezas interpersonales, de administración y liderazgo.

Apoyados de las palabras de Garzón (2018) en su artículo "Uso y apropiación de herramientas TIC de estudiantes y docentes investigadores en universidades en Medellín" que exponen que la globalización ha permitido un nuevo sentido en el empleo de las herramientas TIC, así como una urgencia en aumento asociada a la utilización, como elemento adecuado para realizar las labores de la vida diaria.

En el contexto de la pandemia que se vive actualmente en el mundo, las TIC se han convertido en herramientas de uso obligatorio para efectuar todo tipo de actividades, principalmente en las instituciones educativas de todos los grados académicos, puesto que ha sido un sector al que se le ha considerado vulnerable, manteniéndolo en un trabajo virtual, aportando así a la educación y a la investigación. Definitivamente, la intención es que los alumnos obtengan las competencias investigativas suficientes para favorecer los procesos de investigación en los que participe durante su estancia en la IES a la cual esté adscrito y generar con ello mentes pensantes que logren innovar en diversas áreas de la vida.

\section{REFERENCIAS}

Acuña G., L. A. (2018). Formación de investigadores educativos en Chiapas: realidades y falacias. Revista Latinoamericana de Estudios Educativos (México) [en linea]. XLVIII(1), 103-142[fecha de Consulta 29 de Octubre de 2020]. ISSN: 0185-1284. Disponible en: https://www.redalyc.org/articulo.oa?id=27057919009

Aldana de Becerra, G. M., Castro M. S. P. (2017). Convergencias y divergencias en la apropiación de la formación investigativa: el caso de dos programas académicos colombianos. Revista Virtual Universidad Católica del Norte, 50, 62-80. Recuperado de http://revistavirtual.ucn.edu.co/index.php/RevistaUCN/article/view/813/1331

Banco Mundial (2020). Gasto en investigación y desarrollo (\% del PIB). Disponible en https://datos.bancomundial.org/indicator/GB.XPD.RSDV.GD.ZS. Visitado el 29 de octubre del 2020. 
Bolatovna A., D.; Zhayakovna A., A.; Ivanova B., E.; Murphy, A. y Budanovna A., A. (2016). Forming Master's Degree Students' ICT Competencies as Future Researchers and Educators: a Kazakhstan Case Study. International Journal of Environmental \& Science Education, 11(17), pp. 11175-11218. Recuperado de: https:// files.eric.ed.gov/fulltext/EJ1120590.pdf

Campos C., H. y Ramírez S., M. Y. (2018). Las TIC en los procesos educativos de un centro público de investigación. Apertura, 10(1), pp. 56-70. Recuperado de: http://www.udgvirtual.udg.mx/apertura/index.php/apertura/article/view/1160

Carrasco, S., Baldivieso, S., Di Lorenzo, L. (2016). Formación en investigación educativa en la sociedad digital. Una experiencia innovadora de enseñanza en el nivel superior en el contexto latinoamericano. REDRevista de Educación a Distancia. Núm. 48. Artic. 6. 30-Ene-2016. DOI: 10.6018/red/48/6 http://www.um.es/ead/red/48/selin_et_al.pdf

Cifuentes, J. y Pedraza, J. (2017). Importancia de la investigación en la formación de estudiantes en la modalidad a distancia. En Revista Educación y Humanismo, 19(32), 31-52. http://dx.doi.org/10.17081/eduhum.19.32.2530

Cruz P., M.A.; Pozo V., M.A.; Aushay Y., H.R. y Arias P., A.D. (2019). Las Tecnologías de la Información y de la Comunicación (TIC) como forma investigativa interdisciplinaria con un enfoque intercultural para el proceso de formación estudiantil. e-Ciencias de la Información, 9(1). doi: https://doi.org/10.15517/eci.v1i1.33052

Díaz C., A.V., Sánchez A., R. E. y Rosales R., B. (2018). Metodologías e indicadores académicos, económicos, sociales y tecnológicos para la evaluación del impacto de la investigación científica universitaria. Nexo Revista Científica / Vol. 31, No. 02, pp. 74-88 / Diciembre 2018

Durand-Villalobos, J. P. (2017). Factores que inciden en el desempeño de los grupos de investigación: tres casos de estudio de la Universidad de Sonora. Revista mexicana de investigación educativa, vol. 22, núm. 75, Octubre-Diciembre, 2017, pp. 1143-1167 Consejo Mexicano de Investigación Educativa A.C.

Escalante F., A. E y García P., L. E. (2016). La formación de investigadores en el posgrado en México: ¿una tarea solo del docente?. EDETANIA 50 [Diciembre 2016], 159-173, ISSN: 0214-8560

Estrada, F.M.L.; Quiñónez, E.S. y Pantoja, J.P. (2017) El docente universitario como promotor del pensamiento crítico, competencia del investigador. Aula de Encuentro, volumen 19, no 2, pp. 58-75

Flores O., J. M. (2018). Retos y contradicciones de la formación de investigadores en México. Educar em Revista, Curitiba, Brasil, v. 34, n. 71, p. 35-49, set./out. 2018

Garzón C., L. (2018). Uso y apropiación de herramientas TIC de estudiantes y docentes investigadores en universidades en Medellín. Revista Psicoespacios, Vol. 12, N. 20, pp.49-73. Disponible en https://doi.org/10.25057/issn.2145-2776

George R., C. E. y Salado R., L. I. (2019). Competencias investigativas con el uso de las TIC en estudiantes de doctorado. Apertura, 11(1), pp. 40-55. http:// dx.doi.org/10.32870/Ap.v11n1.1387

Gómez-Vargas, M., García A., M., (2015). Factores influyentes de la gestión del conocimiento en el contexto de la investigación universitaria. Información, cultura y sociedad: revista del Instituto de Investigaciones Bibliotecológicas, núm. 33, diciembre, 2015, pp. 29-46. Universidad de Buenos Aires. Buenos Aires, Argentina. 
González G., R. J. (2019). La formación de jóvenes investigadores en Alemania y México, un estudio comparativo. Educación y ciencia, 8(51), 41-47. ISSN 2448-525X

González, O. A. M. (2019), Alternativas para la formación de investigadores en educación. En D. M. Arzola Franco (coord). Procesos formativos en la investigación educativa. Diálogos, reflexiones, convergencias y divergencias (pp. 129-140). Chihuahua, México: Red de Investigadores Educativos Chihuahua.

Guerrero H., V.; Lagunes D., A.; Torres G.; y C.A., Lau N., J. (2019). Propuesta de semilleros de investigación para el desarrollo de la competencia investigadora en ingenierías. Nexo Revista Científica / Vol. 32, No. 01, pp. 13-26/Junio 2019

Jiménez C., V. E. (2018). La formación de investigadores en la Universidad. ACADEMO (Asunción). Revista de Investigación en Ciencias Sociales y Humanidades. ISSN 2414-8938. Vol. 5 Nro. 1. p. 1-2.

Landeros-Olvera E.; Yáñez-Lozano Á.; Sánchez-Grovas R.; Tzontecomani-Rivera A. (2018). Rompiendo paradigmas en la formación de jóvenes investigadores estudiantes de enfermería Rev Enfer Neurol 2018;17(3):pp 29-38.

Lozoya M., E. y Ocampo R., E. A. (2019). Estrategias para la formación de investigadores en investigación educativa. En D. M. Arzola Franco (coord). Procesos formativos en la investigación educativa. Diálogos, reflexiones, convergencias y divergencias (pp. 141-174). Chihuahua, México: Red de Investigadores Educativos Chihuahua.

Martínez M., S.; Medina P., F.;y Salazar C., L. (2018). Desarrollo de competencias investigativas en los estudiantes. Opuntia Brava, 10(1), 336-341. https://doi.org/https://doi.org/10.35195/ob.v10i1.80

Olivares, K.; Angulo, J.; Torres, C. y Madrid, E. (2016). Las TIC en educación: metaanálisis sobre investigación y líneas emergentes en México. Apertura, 8, (2). pp. 100-115. doi: http://dx.doi.org/10.32870/Ap.v8n2.866

Parra-Castrillón, J. E. (2018), Construcción de la competencia investigativa en ingeniería, Revista Educación en Ingeniería, 13(25), pp. 12-19, Febrero, 2018, Bogotá. ISSN 1900-8260 DOI: http://dx.doi.org/10.26507/rei.v13n25.812

Pastor R., D.; Arcos M., G. L. y Lagunes D., A. (2020). Desarrollo de capacidades de investigación para estudiantes universitarios mediante el uso de estrategias instruccionales en entornos virtuales de aprendizaje. Apertura, 12(1). http://dx.doi.org/10.32870/Ap.v12n1.1842

Pedraza L., J. S. (2018) Experiencias de formación como investigadores educativos de estudiantes de un programa de doctorado en educación. Actualidades Investigativas en Educación, vol. 18, núm 2, MayoAgosto, 2018, pp. 1-33. Instituto de Investigación en Educación, Universidad de Costa Rica, DOI: 10.15517/aie.v18i2.33134

Pepper-Loza, K. F. y Terán-Vega, J. D. (2019). El semillero de investigación estudiantil, como estrategia para la Formación de investigadores. Polo del Conocimiento, (Edición núm. 39) Vol. 4, No 11, pp. 4-20 ISSN: 2550 - 682X

Pérez, A. D. (2017). Formación para la investigación y efciencia terminal. Una tensión en los posgrados en Educación. Libro Formación para la investigación en los posgrados en educación. Perfles, Trayectorias, experiencias y saberes. Toluca, ISCEEM 
Ramírez, N. (2015). Ansiedad bibliotecaria en estudiantes universitarios. Revista Interamericana de Bibliotecología, 38(3), 227-236. doi: 10.17533/udea.rib.v38n3a07

Redcolsi (2016), Seguimos creciendo, [Internet] disponible en <http://fundacionredcolsi.org/web/index.php/2- uncategorised/92-about-us $>$.

Rojas, M. y Méndez, R. (2017) Procesos de formación en investigación en la Universidad: ¿Qué le queda a los estudiantes? Sophia 13 (2): 53-69. DOI: http://dx.doi.org/ 10.18634/sophiaj.13v.2i.261

Rojas S., J. A. (2018). Héroes del futuro: Desafío para la formación de investigadores con sensibilidad social. En Bautista Pinzón, M. A., Castellanos Martínez, Y. V., Cipagauta Moyano, M. E., Experiencias en el aula. Tercer encuentro de prácticas pedagógicas innovadoras. Pag. 45-52, Corporación Universitaria Minuto de Dios - UNIMINUTO, Bogotá, 2018. ISSN digital: 2619-3132

Ruiz M., Y. A. (2019). Evaluación formativa compartida para el desarrollo de competencias investigativas en estudiantes universitarios. Educere [en línea]. 2019, 23(75), 499-508 [fecha de consulta 21 de octubre de 2020]. ISSN: 1316-4910. Disponible en: https://www.redalyc.org/articulo.oa?id=35660262020

Troncoso, C.P.C., Rojas R. E., Diosdado. E.P. (2014). Cómo transformar al alumno en investigador. Pistas Educativas No. 110, 129-136.

Vega-Monsalve, N. (2019), "Estrategias de conformación y consolidación de semilleros de investigación en pregrado. Estudio de caso en una institución de educación superior en Colombia", en Revista Iberoamericana de Educación Superior (ries), México, unam-iisue/Universia, vol. x, Núm. 27, pp. 216-229, doi: dx.doi.org/10.22201/ iisue.20072872e.2019.27.344 [consulta: 19 de octubre de 2020].

\section{SEMBLANZA DE LOS AUTORES}

Rosario Carvajal-Hernández: Obtuvo el grado de Licenciada en Informática en el Instituto Tecnológico Superior de San Andrés Tuxtla, Veracruz, México, donde actualmente se desempeña como docente. Desarrolló sus estudios de maestría en la Universidad Cristóbal Colón en Veracruz, México. Ha participado en trabajos de investigación en la línea de investigación "Desarrollo de soluciones basadas en tecnologías emergentes", así como en el Cuerpo Académico en formación "Aplicabilidad Informática en ámbitos heterogéneos". Reconocimiento de Perfil Deseable PRODEP desde 2014 a la fecha.

Verónica Guerrero-Hernández: Maestría en Tecnologías de Información por la Universidad Cristóbal Colon de Veracruz, Ver. México y Licenciada en Informática por el Instituto Tecnológico de Orizaba, México. Profesora de tiempo completo en el Instituto Tecnológico Superior de San Andrés Tuxtla desde 1997. Reconocimiento de Perfil Deseable PRODEP 2020. Trabaja en líneas de investigación vinculadas con el desarrollo de tecnologías emergentes.

Erick de Jesús Téllez-Vera: Maestría en Tecnologías de Información por la Universidad Cristóbal Colon de Veracruz, Ver. México y Licenciada en Informática por el Instituto Tecnológico de Orizaba, México. Profesor de tiempo completo en el Instituto Tecnológico Superior de San Andrés Tuxtla. Reconocimiento de Perfil Deseable PRODEP vigente. Trabaja en líneas de investigación vinculadas con el desarrollo de tecnologías emergentes. 